\title{
CONSISTENCY ISSUES IN HAIR SPECTRAL REFLECTANCE AND COLOR MEASUREMENTS
}

\author{
Simone Liberini ${ }^{1}$, Antonio Avolio ${ }^{2}$, Stevn Thomas Yankowski ${ }^{3}$, Alessandro Rizzi ${ }^{1}$ \\ ${ }^{1}$ Dipartimento di Informatica, Università degli Studi di Milano, Milan (Italy); \\ ${ }^{2}$ Universal Beauty Products, Brescia (Italy); \\ ${ }^{3}$ BIG Professional, Brescia (Italy)
}

\section{ABSTRACT}

The color shade quality control scenario is ubiquitous within the cosmetic hair coloring industry. Permanent hair dyes are an especially marketable product, so their diffusion is dependent on stringent quality controls that guarantee product safety and adherence to expected results. To this day, visual inspection is the go-to standard for the evaluation of dye applications outcomes, even though the increased availability of easily operated spectrophotometers has favored the introduction of colorimeters in the manufacturing pipeline. Human hairs, however, are very efficient scatterers ${ }^{1}$ and this makes their instrumental measurement more difficult and less consistent. In order to test the degree to which this intrinsic property compromises the reliability of spectrophotometric measurements, we tested a variety of samples of human hair locks (swatches), both dyed and undyed, and those of nylon fiber hair swatches.

First, we analyzed results from two measuring modalities made available by instrumentation: Specular Component Included (SCI) and Specular Component Excluded (SCE). Then, in order to modify macroscopic spatial orientation, we arranged swatches so that hair strands would cross at varying angles. Measurements confirmed the expected dependencies, namely the clear influence of the scattered component and of sample orientation on instrumental reading outputs.

Having settled on a preferred measuring setup, we analyzed measurement variance. Again, we evaluated two specific aspects, which tie directly into industrial quality assessments. We briefly discussed qualitative visual inspection of spectra. We then switched to $\Delta$ Es to show the unreliability, at least as far as lighter colored dyes are concerned, of classic target-sample measurements employed to issue pass-or-fail verdicts.

Despite quantitative measurements being an asset for quality control, we suggest that greater care is placed in spectrophotometry readings when human hair is involved, as opposed to more traditional fields of application.

Keywords: Human hair, Cosmetology, Spectrophotometry.

\section{INTRODUCTION}

The outer portion of human hair, the shaft, is composed of three main concentric layers (figure 1). Its innermost core, the medulla, is a discontinuous lipidic substrate, rich in mitochondrial DNA. On the outside and concentrically lies the cortex, a layer of longitudinal cells making up for the vast majority of the total weight of the shaft. These cells confer hair most of their mechanical properties, and the shape of their cross section makes them appear straight, wavy or curly ${ }^{3}$. Finally, the outer portion, the cuticle, provides hair their structural integrity and water retention within the innermost layers. 


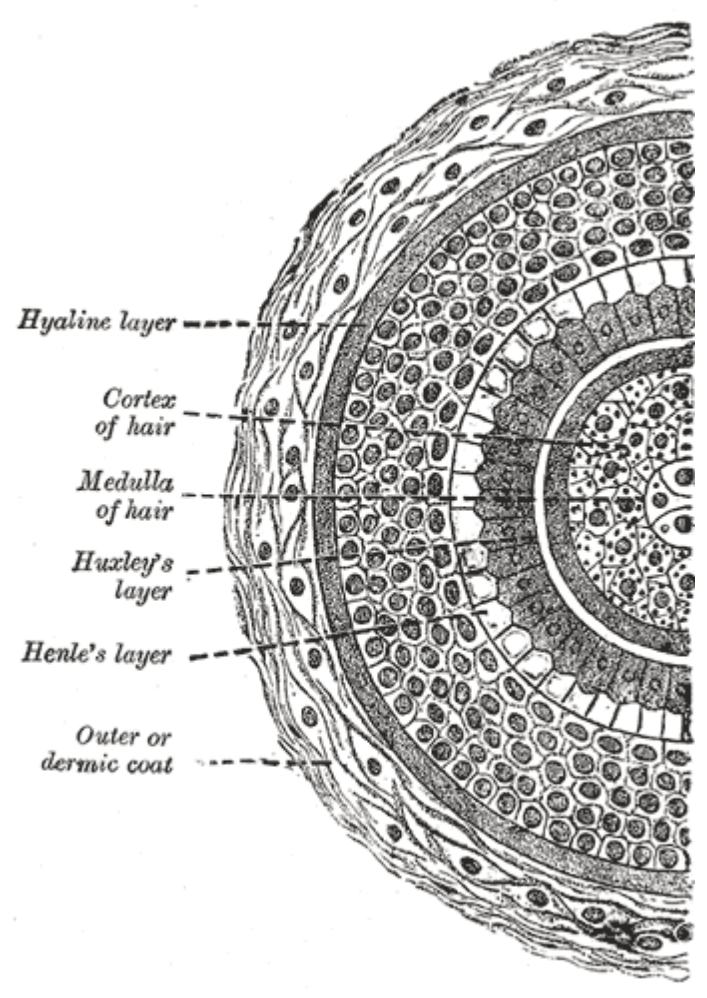

Figure 1. Cross section of human hair, from Anatomy of the Human Body by Henry Gray (1918)

Cortical cells are also home to melanin, a natural polymer whose production entails a complex pathway of highly unstable intermediates synthesized within melanocytes, contained in melanosomes and ultimately uptaken by keratinocytes within the shaft. Two main varieties of melanin exist, both responsible for hair pigmentation. Higher concentrations of eumelanin prevail in black and brown hair, whereas low concentrations in blond hair. On the contrary, a prominence of pheomelanin results in red hair. The strikingly wide gamut of intermediate colors occurring naturally in human head hair is the product of varying mixtures of the two, though shape and size of melanosomes also contribute to the final overall appearance ${ }^{3}$.

Natural hair color has long been tampered with in the history of humankind. Hair dyes represent, as of today, a flourishing market segment within the cosmetic industry, the vast majority of dyes sold worldwide being permanent in that they guarantee color retention even after multiple washes. High durability is attained through a combination of chemicals ${ }^{3}$. Molecules conferring hair the desired final color, still absent at the time of application, are let through to the inner layers by means of an alkaline substance - the likes of ammonia-which raises $\mathrm{pH}$ to the point where the cuticle opens up along the intricate weave of natural slits slanting over its outermost surface. Melanin bonds are then dissolved by a strong oxidizing agent, such as hydrogen peroxide, which dissolves and removes natural pigmentation. The strong oxidizing environment is also responsible for the initiation of the dyeing process from deep within the cortex, where aromatic amines react with coupling agents that, typically in a 30 to 45 minutes timespan, will produce the final color and seal it tightly within the cortex through a barrier of high molecular weight stable compounds.

Hair structure natural variability, leftovers of previously applied dyes, and general failure to abide by instructions for use may alter the desired outcome of dye applications, so a good deal of expertise on behalf of practitioners is often required to avoid mishaps. In addition, environmental conditions such as air humidity and temperature may contribute to undesired results, so that a controlled setting is ideal in which to operate. It is, however, of utmost importance for dye manufacturers to guarantee their products both in terms of substance stability and output consistency. The addition of supplementary reactants is meant to secure reasonable shelf-life and safety of use. As 
far as meeting expectations upon application goes, it is customary that practitioners visually inspect end results. In industrial settings, in addition, it is not uncommon to also employ spectrophotometric measurements to produce quantitative assessments of reliability. These measurements usually output spectral plots to be evaluated over the visible range, from which CIE L*a*b* triplets can be synthesized to compute $\Delta \mathrm{Es}^{4}$. Arguably though, these pieces of information should be treated with some degree of caution.

Due to inherent optical density, and to differences in material composition, structure, and cross-section size, human hairs are very efficient scatterers ${ }^{1}$. The type of scattering is highly dichroic (i.e. polarization sensitive) because of hair innermost structures' natural anisotropy. Measurements of hair samples are consequently highly dependent on the geometrical configuration of the measuring setup - orientation of the sample, angle of incidence, polarization of the light, aperture of the system, and perhaps most importantly, color, i.e. the light absorption factor which is a further channel of extinction of light.

We hereby present tests aiming at verifying the possibility of assessing hair color with spectral instruments, analyzing and discussing the variability of measurements and the influences of the measuring setup.

\section{MATERIALS AND METHODS}

\subsection{Real hair swatches}

Hair samples came in the shape of swatches around $1.5 \mathrm{~g}$ in weight, folded about their midpoint and tightly secured by the loop of a plastic zip tie (figure 2a). Two such specimens were arranged for the following undyed swatches: yak coat hair, chestnut brown human hair, and the three mixtures 80/20, 50/50, 20/80, numbers to the left representing the percentage of white hair strands over the total, as opposed to pigmented strands. Two specimens were also prepared of these five swatch types by dyeing them Cold Black (1.0), Cold Dark Blond (6.0) and Ash Platinum Blond (10.1), for a grand total of 40 samples. All swatches were dyed in a controlled environment, at $20^{\circ} \mathrm{C}$, with a 20 volume developer mixed in a ratio of 1 to 1.5 . Dyes were applied lengthwise in both directions to ensure maximum coverage and penetration, then left to process for 30 minutes. After being dyed, hair was properly washed, rinsed and an adequate dose of hair conditioner was applied, to better mimic results obtained in a hairdresser's salon and to bring cuticles back to a physiologically sealed state. No other cosmetic products, such as hairspray, were applied after the process.

\subsection{Nylon hair swatches}

A set of nylon swatches was also measured. These were taken from the official color sampler of a renowned brand, selected by visual inspection of a varied amount of testers at our disposal. Each sample is a handful of dyed nylon strands, glued at both ends, in turn glued together into a horseshoe shape (figure $2 \mathrm{~b}$ ). The 10 swatches that were analyzed are those identified by the manufacturer as the "natural" series, the one, that is, supposedly mimicking a selection of color naturally occurring in human hair: Black (1), Deep Dark Chestnut Brown (2), Dark Chestnut Brown (3), Chestnut Brown (4), Light Chestnut Brown (5), Dark Blond (6), Blond (7), Light Blond (8), Very Light Blond (9) and Platinum Very Light Blond (10). Swatches were gently ripped form the cardboard support, kept in the shape they came from and did not undergo any kind of further treatment.

\subsection{Instrumentation}

Measurements were taken with a Konica Minolta CM-2600d spectrophotometer, then collected and inspected with the interfacing software Spectramagix NX. Among available parameters, no ultraviolet cutoff and a $8 \mathrm{~mm}$ diameter mask (Medium Aperture Value, MAV) were selected. Spectral plots were acquired and charted from the minimum wavelength $360 \mathrm{~nm}$ to the maximum wavelength $740 \mathrm{~nm}, 10 \mathrm{~nm}$ stepwise, and for CIE $\mathrm{L}^{*} \mathrm{a}^{*} \mathrm{~b}^{*}$ calculations, CIE $2^{\circ}$ standard observer and a D50 illuminant were selected. 
In order to test the scattering efficiency of hair, both Specular Component Included (SCI) and Specular Component Excluded (SCE) measurements were acquired and analyzed. Smooth surfaces contribute a conspicuous amount of specular reflection, such that incident rays will travel in a shapely conformation out of the surface and towards the observer, who will experience the appearance of gloss. On the contrary, rough surfaces will bounce off incident light rays in a disorderly fashion, so that the observer will perceive them as matte. Not only surface roughness contributes to gloss perception, it also influences color perception as well. In fact, high-gloss surfaces will usually appear more saturated, their color more vivid, while matte surfaces will look duller, their color weaker.

\subsection{Measuring setups}

A white paper sheet was placed beneath hair swatches to evaluate and rule out the possibility of background interferences. Its spectrum shows a high-reflectance plateau from medium to high wavelengths, and a prominent peak at low wavelengths due to treatments with optical brighteners. This conspicuous characteristic would have led to visible spectrum perturbations had the white background seeped through hair strands.

Two measurement setups were arranged in order to evaluate variance. Each swatch of hair was sampled five times along its entire length on both sides, starting a little distance from the tightening loop and moving regularly towards the loose end down to a safe margin from hair tips. Having two equal specimens for every kind of human hair swatch, a grand total of twenty samples per swatch type was acquired. Each nylon swatch was instead granted half such measurements.

Also for human hair, a second batch of measurements was taken by keeping one swatch fixed in place and superimposing its twin swatch spread in a fanned arrangement, obtained by pinching it near the zip tie loop by means of a metal clip (figure 2c). This allowed the evaluation of measurement variations due to hair strands slanting at different angles with respect to a fixed direction. Five measurements were taken for each of the four possible combinations of both sides for both swatches, yet again for a grand total of twenty samples per swatch type.
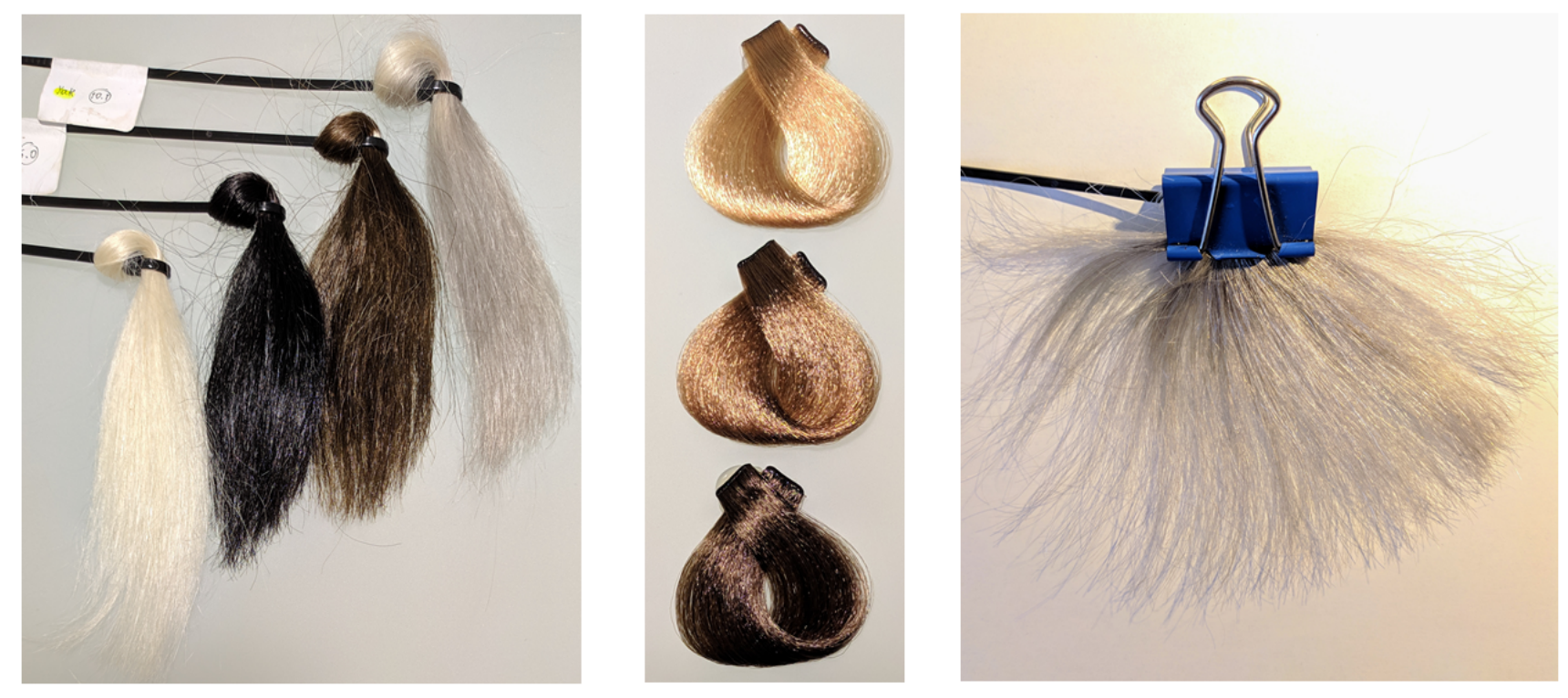

Figure 2. (a) Hair swatches (Yak undyed, Yak 1.0, Yak 6.0, Yak 10.1); (b) Nylon swatches (very light blond, blond, chestnut brown); (c) Fanned hair for crossed setup measurements. 


\section{RESULTS}

For any given hair swatch, four possible versions exist that reflect the combinations between SCI or SCE and the lengthwise or crossed arrangement-for instance Yak 6.0 SCI (SCE) Lengthwise (Crossed). Before assessing measurement variability per se, we proceed with a preliminary assessment of the effect of specular component.

\subsection{SCI versus SCE}

SCI and SCE for all five swatch types, both dyed and undyed and sampled lengthwise, are shown in figure 3. Solid lines are the means over 20 spectral samples, each surrounded by a symmetric band delimited by dashed lines representing standard deviations. At a first glance, graphs from (a) to (e) appear to depict only four lines each, due to the SCI and the SCE variants being nearly indistinguishable one from the other. A magnification is portrayed in (f) for brown swatches. As check and also to give to the reader a visual idea, an SCI versus SCE plot of a glossy magazine cover is reported in figure 4 , whose difference is on the contrary readily appreciable.

An agreed standard method to assess spectral difference does not exist. In order to give an example of this problem, the $\mathrm{L}_{1}$ norm between mean spectra- one possible quantitative correlate of the qualitative idea of "spectral closeness"-is reported in Table 1 and 2, together with the relative $\Delta$ Es. As it can be noticed from the values, $\mathrm{L}_{1}$ norm is not predictive of color difference.
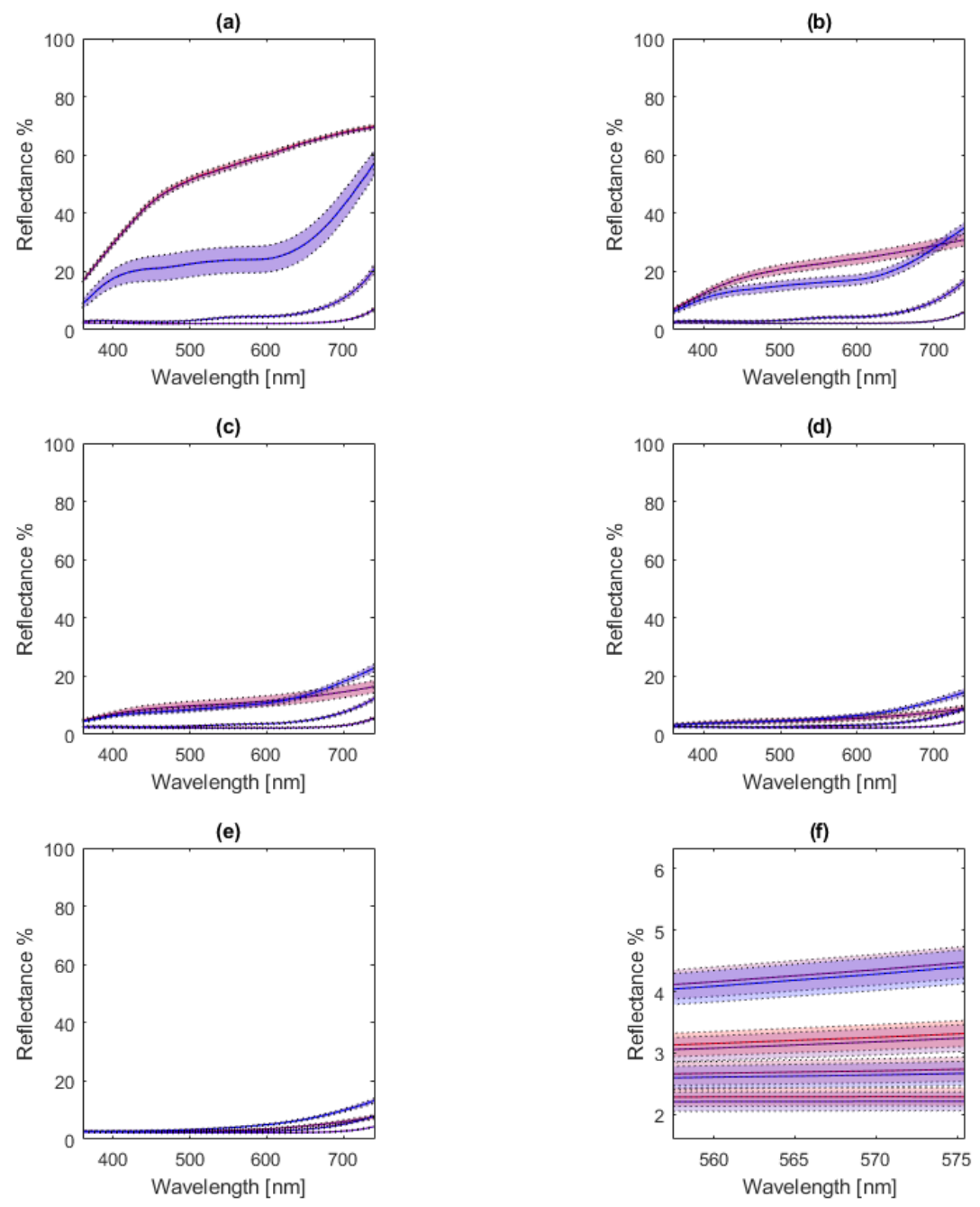

Figure 3. SCI and SCE variants of human hair swatches. (a) Yak; (b) 80/20; (c) 50/50; (d) 20/80; (e) chestnut brown;

(f) chestnut brown magnification, in which subtle differences between SCI and SCE are visible. 


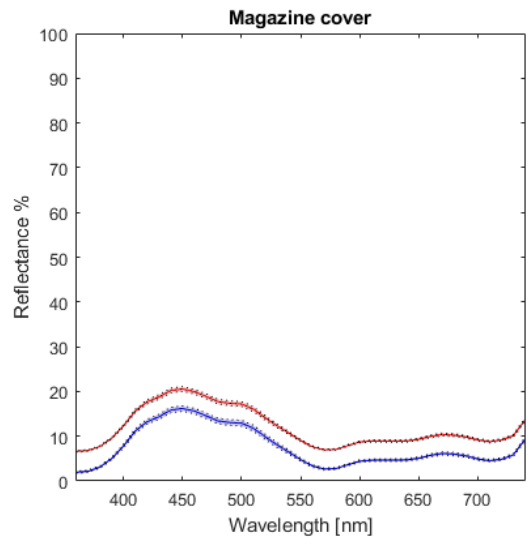

Figure 4. SCI and SCE variants of a glossy magazine cover.

Table $1 . \mathrm{L}_{1}$ norm and $\Delta \mathrm{E}$ - SCI versus SCE, human hair swatches.

\begin{tabular}{lccccccccc} 
& \multicolumn{2}{c}{ Undyed } & \multicolumn{2}{c}{1.0} & \multicolumn{2}{c}{6.0} & \multicolumn{2}{c}{10.1} \\
& $\mathrm{~L}_{1}$ & $\Delta \mathrm{E}$ & $\mathrm{L}_{1}$ & $\Delta \mathrm{E}$ & $\mathrm{L}_{1}$ & $\Delta \mathrm{E}$ & $\mathrm{L}_{1}$ & $\Delta \mathrm{E}$ \\
Yak & 8.0390 & 0.1493 & 2.5260 & 0.3152 & 1.5330 & 0.2276 & 3.3515 & 0.1127 \\
$80 / 20$ & 1.8030 & 0.0887 & 2.4720 & 0.3122 & 2.3385 & 0.2781 & 1.4240 & 0.0907 \\
$50 / 50$ & 1.5330 & 0.1161 & 3.3280 & 0.4300 & 2.6405 & 0.3102 & 2.0280 & 0.1581 \\
$20 / 80$ & 2.9775 & 0.2480 & 3.2070 & 0.4239 & 3.3805 & 0.3963 & 2.3365 & 0.2374 \\
Brown & 2.6730 & 0.3488 & 2.7975 & 0.3748 & 2.3900 & 0.3164 & 2.7135 & 0.3123
\end{tabular}

Table 2. $\mathrm{L}_{1}$ norm and $\Delta \mathrm{E}-\mathrm{SCI}$ case versus $\mathrm{SCE}$ case, nylon swatches, mean spectra.

$\begin{array}{ccccccccccc} & 1 & 2 & 3 & 4 & 5 & 6 & 7 & 8 & 9 & 10 \\ \mathrm{~L}_{1} & 9.0960 & 13.1660 & 15.9750 & 16.7200 & 18.2960 & 24.5170 & 25.6590 & 37.3130 & 42.7620 & 62.0100 \\ \Delta \mathrm{E} & 0.6357 & 0.7041 & 0.8203 & 0.8417 & 0.8119 & 1.1661 & 1.1659 & 1.4829 & 1.3334 & 1.5179\end{array}$

Analyzing $\triangle \mathrm{E}$, SCI and SCE measurements result nearly identical, as no combination of hair swatch and dye ever approaches the value of 1 (median value 0.2942). On the other hand, the same does not necessarily hold true for nylon swatches. In this case, as per Table 2, bottom row, $\Delta$ Es are generally higher (median value 1.0038), so a difference exists between SCI and SCE that the human eye would also able to perceive. In fact, nylon strands are more homogenous, their texture and spatial disposition more regular and smoother, the material perceivably glossier than human hair. This behavior is exacerbated when nylon swatches are observed from a tilted perspective, when patches of perceivably different color stand out even more.

\subsection{Lengthwise versus Crossed}

When SCI Lengthwise versus Crossed dispositions are considered, the minimum threshold $\Delta \mathrm{E}=1$ is exceeded 12 out of 20 times (Table 3), and $\triangle E$ Es are overall higher than the SCI versus SCE case (median value 1.1214). This confirms the influence of the swatch spatial arrangement under the instrument. Difference in $\mathrm{L}_{1}$ norm and $\Delta \mathrm{E}$ are reported in Table 3. $\Delta \mathrm{E}$ ranges from 0.52 to 3.10 , which amounts to a significant color difference especially considering it derives from the swatch spatial arrangement under the instrument alone. 
Table 3. $\mathrm{L}_{1}$ norm and $\Delta \mathrm{E}$ - Lengthwise case versus Crossed case, human hair.

\begin{tabular}{lcccccccc} 
& \multicolumn{2}{c}{ Undyed } & \multicolumn{2}{c}{1.0} & \multicolumn{2}{c}{6.0} & \multicolumn{2}{c}{10.1} \\
& $\mathrm{~L}_{1}$ & $\Delta \mathrm{E}$ & $\mathrm{L}_{1}$ & $\Delta \mathrm{E}$ & $\mathrm{L}_{1}$ & $\Delta \mathrm{E}$ & $\mathrm{L}_{1}$ & $\Delta \mathrm{E}$ \\
Yak & 63.0745 & 1.0818 & 7.3190 & 0.8205 & 9.7560 & 0.5210 & 133.8055 & 3.1045 \\
$80 / 20$ & 47.7380 & 1.3331 & 6.2050 & 0.6057 & 21.5430 & 1.7192 & 53.2420 & 1.6816 \\
$50 / 50$ & 38.2370 & 1.7120 & 9.5395 & 0.9635 & 20.3475 & 1.8289 & 36.2795 & 1.6348 \\
$20 / 80$ & 14.6115 & 0.8616 & 10.0645 & 1.1609 & 11.5665 & 1.0167 & 15.0885 & 0.8101 \\
Brown & 9.7875 & 0.8250 & 8.5655 & 0.9479 & 12.6600 & 1.2479 & 20.2025 & 1.5625
\end{tabular}

\subsection{Results on variance}

We proceed by restricting our analysis to SCI Lengthwise swatches, both for human hair and for nylon. Mean spectra with standard deviation bands, plus upper and lower envelope bands are shown in figure 5, grouped both on a per-swatch and on a per-dye basis for human hair. Spread of chromaticity coordinates are portrayed in figure 6 for human hair, and in figure 7 for nylon swatches for all acquired spectra. Figure 8 shows the portions of the chromaticity diagram occupied by scatterplots in figure 6 and figure 7 .
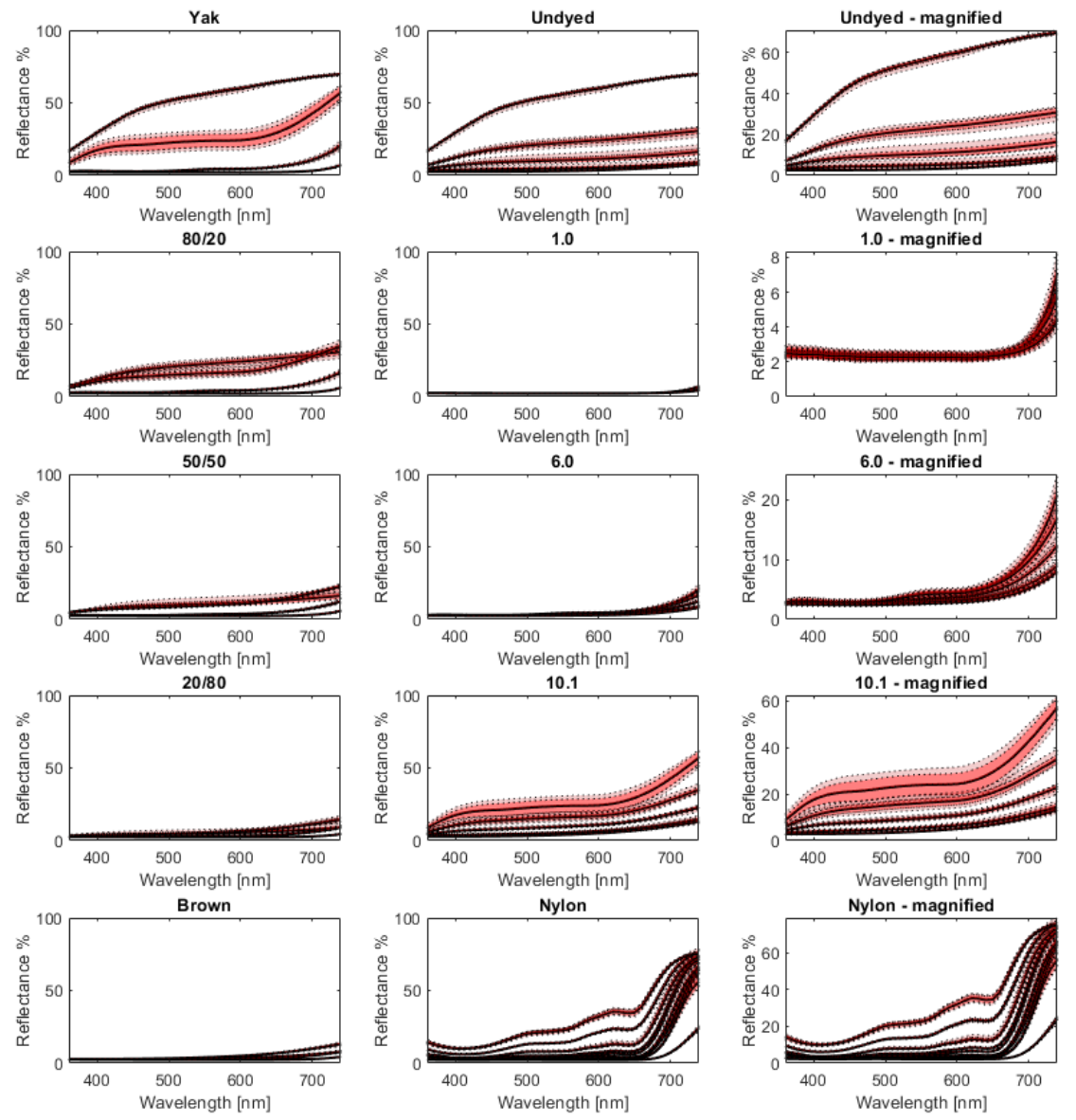

Figure 5. Mean spectra with standard deviation and envelope bands of swatches grouped per swatch (first column) and per dye (second column, third column magnified). 


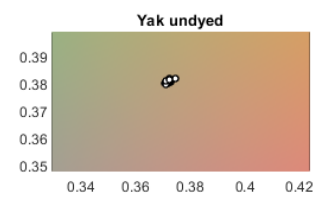

$80 / 20$ undyed

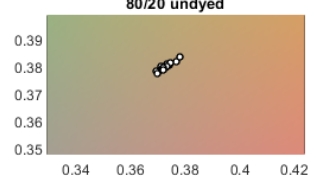

$50 / 50$ undyed

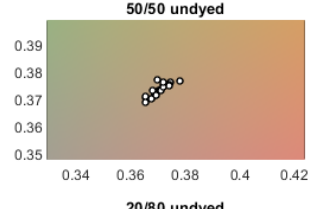

20/80 undyed
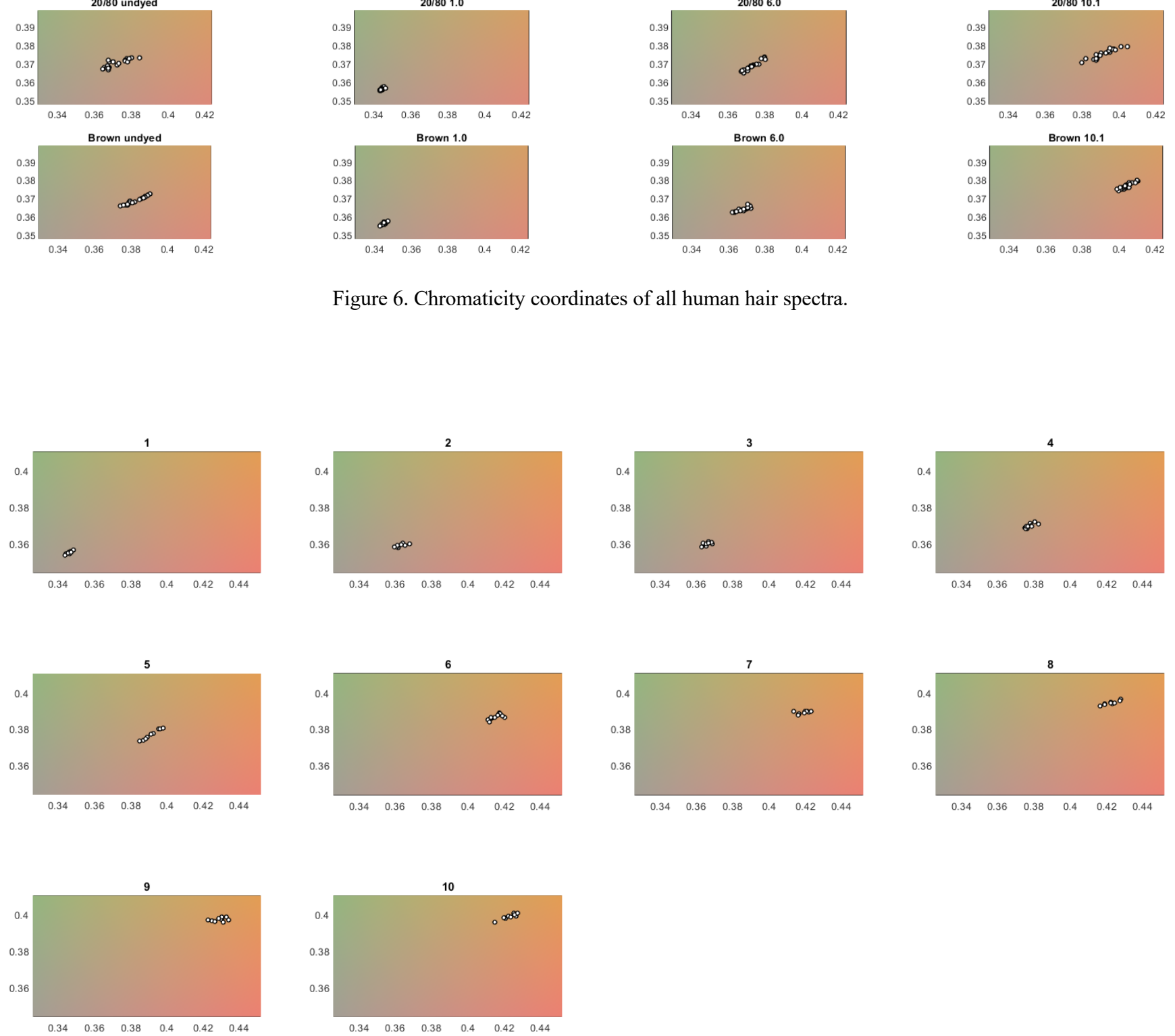

Figure 7. Chromaticity coordinates of all nylon swatches spectra.

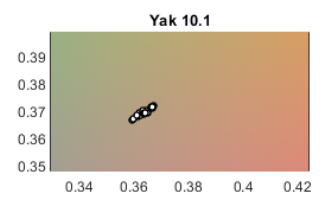

$80 / 2010.1$

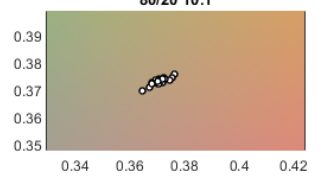

$50 / 5010.1$
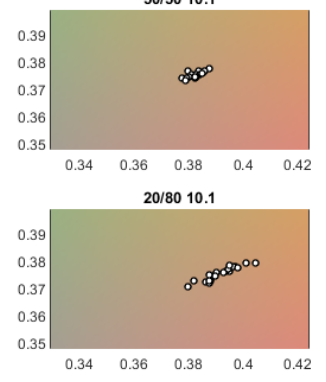

Brown 10.1

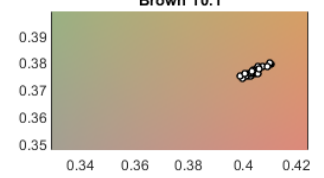

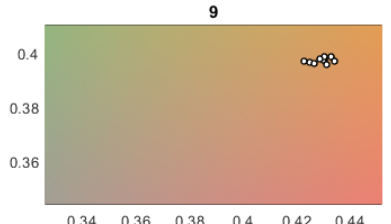




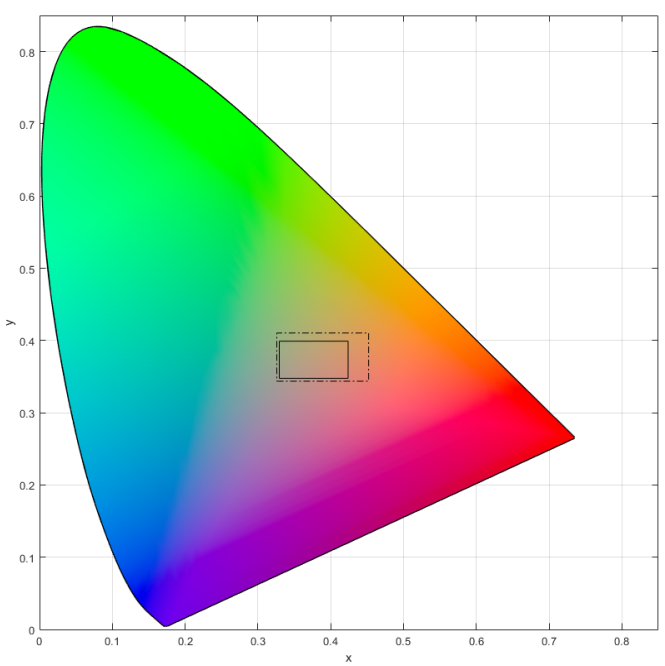

Figure 8 . Portions of the chromaticity diagram occupied by scatterplots in figure 5 (solid line) and figure 6 (dash-dotted line)

Table 4. Means and standard deviations of spectral values, chromaticity coordinates x and y, and CIE Lab.

\begin{tabular}{|c|c|c|c|c|c|c|c|c|c|c|c|}
\hline & $\sigma$ & $\mu_{\mathrm{x}}$ & $\sigma_{\mathrm{x}}$ & $\mu_{\mathrm{y}}$ & $\sigma_{\mathrm{y}}$ & $\mu_{\mathrm{L}}$ & $\sigma_{\mathrm{L}}$ & $\mu_{\mathrm{a}}$ & $\sigma_{\mathrm{a}}$ & $\mu_{\mathrm{b}}$ & $\sigma_{\mathrm{b}}$ \\
\hline Yak undyed & 0.9623 & 0.3722 & 0.0009 & 0.3809 & 0.0006 & 80.05 & 0.61 & 1.85 & 0.23 & 12.80 & 0.32 \\
\hline Yak 1.0 & 0.1316 & 0.3475 & 0.0018 & 0.3581 & 0.0010 & 16.38 & 0.41 & 0.30 & 0.13 & 0.07 & 0.23 \\
\hline Yak 6.0 & 0.4565 & 0.3951 & 0.0030 & 0.3878 & 0.0020 & 24.43 & 1.09 & 3.23 & 0.31 & 8.47 & 0.71 \\
\hline Yak 10.1 & 4.2505 & 0.3636 & 0.0023 & 0.3694 & 0.0015 & 55.94 & 4.53 & 2.12 & 0.20 & 5.34 & 0.53 \\
\hline $80 / 20$ undyed & 1.6517 & 0.3729 & 0.0024 & 0.3800 & 0.0015 & 54.99 & 1.86 & 1.81 & 0.29 & 9.35 & 0.79 \\
\hline $80 / 201.0$ & 0.1340 & 0.3455 & 0.0015 & 0.3567 & 0.0010 & 16.70 & 0.53 & 0.22 & 0.09 & -0.22 & 0.21 \\
\hline $80 / 206.0$ & 0.4668 & 0.3885 & 0.0024 & 0.3826 & 0.0018 & 24.09 & 1.27 & 3.00 & 0.21 & 7.02 & 0.57 \\
\hline $80 / 2010.1$ & 1.6586 & 0.3707 & 0.0028 & 0.3731 & 0.0014 & 47.56 & 2.28 & 2.73 & 0.36 & 6.49 & 0.58 \\
\hline $50 / 50$ undyed & 1.5852 & 0.3705 & 0.0033 & 0.3733 & 0.0024 & 39.12 & 2.84 & 2.29 & 0.34 & 5.69 & 0.96 \\
\hline $50 / 501.0$ & 0.2079 & 0.3456 & 0.0012 & 0.3568 & 0.0009 & 17.07 & 0.77 & 0.22 & 0.10 & -0.21 & 0.18 \\
\hline $50 / 506.0$ & 0.2876 & 0.3816 & 0.0045 & 0.3745 & 0.0028 & 21.93 & 0.79 & 3.03 & 0.32 & 4.97 & 0.80 \\
\hline $50 / 5010.1$ & 0.8211 & 0.3823 & 0.0028 & 0.3752 & 0.0012 & 37.74 & 1.47 & 4.29 & 0.34 & 7.23 & 0.49 \\
\hline 20/80 undyed & 0.8836 & 0.3726 & 0.0059 & 0.3702 & 0.0022 & 27.11 & 2.48 & 2.64 & 0.59 & 4.13 & 0.74 \\
\hline $20 / 801.0$ & 0.1804 & 0.3438 & 0.0008 & 0.3560 & 0.0006 & 17.44 & 0.74 & 0.08 & 0.07 & -0.42 & 0.12 \\
\hline $20 / 806.0$ & 0.2694 & 0.3727 & 0.0042 & 0.3686 & 0.0026 & 20.38 & 0.89 & 2.51 & 0.31 & 3.31 & 0.72 \\
\hline $20 / 8010.1$ & 0.6848 & 0.3920 & 0.0062 & 0.3755 & 0.0026 & 28.86 & 1.58 & 5.18 & 0.54 & 7.05 & 0.99 \\
\hline Brown undyed & 0.2862 & 0.3826 & 0.0050 & 0.3689 & 0.0021 & 21.02 & 0.78 & 3.93 & 0.47 & 4.18 & 0.73 \\
\hline Brown 1.0 & 0.1640 & 0.3449 & 0.0010 & 0.3562 & 0.0006 & 16.94 & 0.73 & 0.20 & 0.09 & -0.32 & 0.13 \\
\hline Brown 6.0 & 0.2265 & 0.3675 & 0.0031 & 0.3639 & 0.0012 & 18.83 & 0.81 & 2.34 & 0.32 & 2.18 & 0.37 \\
\hline Brown 10.1 & 0.3157 & 0.4044 & 0.0032 & 0.3768 & 0.0016 & 24.73 & 0.77 & 6.38 & 0.30 & 7.77 & 0.60 \\
\hline
\end{tabular}


In order to assess variability, two kinds of computations were made: intra-swatch, i.e. within a single swatch, and inter-swatch, i.e. between pairs of swatches of the same type and dye. Both are based on the calculation of $\Delta \mathrm{E}$ among all the spectrophotometric measurements acquired and detailed in subsection 2.4. In the intra-swatch case, both the first point and the second among which $\Delta \mathrm{E}$ is evaluated belong to the same swatch of hair. Having two swatches per type and dye, mean values are calculated. In the inter-swatch case, the first point belongs to one out of any two given swatches, and the second point to the corresponding swatch. All possible combinations of points are considered: for intra-swatch a total of 45 cases per swatch; for inter-swatch, 100. Maximum, mean and minimum $\Delta$ Es are calculated. Results are shown in Tables 5 for human hair and for nylon swatches.

Table 5. $\Delta$ Es - Human hair intra-swatch (left), human hair inter-swatch (center), nylon intra-swatch (right)

\begin{tabular}{|c|c|c|c|c|c|c|c|c|c|c|c|c|c|}
\hline & $\Delta \mathrm{E}_{\min }$ & $\Delta \mathrm{E}_{\mathrm{m}}$ & $\Delta \mathrm{E}_{\max }$ & $\sigma_{\Delta \mathrm{E}}$ & $\Delta \mathrm{E}_{\min }$ & $\Delta \mathrm{E}_{\mathrm{m}}$ & $\Delta \mathrm{E}_{\max }$ & $\sigma_{\Delta \mathrm{E}}$ & & $\Delta \mathrm{E}_{\min }$ & $\Delta \mathrm{E}_{\mathrm{m}}$ & $\Delta \mathrm{E}_{\max }$ & $\sigma_{\Delta \mathrm{E}}$ \\
\hline Yak Undyed & 0.1479 & 0.8645 & 2.2074 & 0.5165 & 0.1212 & 0.8908 & 2.5968 & 0.5329 & 1 & 0.1067 & 1.1591 & 2.6180 & 0.7228 \\
\hline Yak 1.0 & 0.0822 & 0.5599 & 1.3850 & 0.2945 & 0.0675 & 0.6697 & 1.5517 & 0.2806 & 2 & 0.1922 & 1.0569 & 2.3162 & 0.5758 \\
\hline Yak 6.0 & 0.3159 & 1.6346 & 4.1405 & 0.9320 & 0.1329 & 1.6594 & 4.3626 & 0.9107 & 3 & 0.2922 & 1.1002 & 2.5211 & 0.6053 \\
\hline Yak 10.1 & 0.5332 & 2.8510 & 7.9655 & 1.8774 & 0.8787 & 7.7257 & 13.8586 & 3.0239 & 4 & 0.3509 & 1.1026 & 2.3955 & 0.5345 \\
\hline 80/20 Undyed & 0.2023 & 2.3372 & 5.7724 & 1.2888 & 0.4626 & 2.6899 & 7.1360 & 1.4675 & 5 & 0.2008 & 1.3296 & 2.7261 & 0.6290 \\
\hline $80 / 201.0$ & 0.1315 & 0.7110 & 1.8324 & 0.3872 & 0.0242 & 0.6883 & 1.8135 & 0.4070 & 6 & 0.2873 & 1.4312 & 2.8351 & 0.6559 \\
\hline $80 / 206.0$ & 0.2656 & 1.5893 & 4.1051 & 0.9115 & 0.1516 & 1.8355 & 4.8822 & 1.0978 & 7 & 0.0882 & 1.2044 & 2.5821 & 0.6077 \\
\hline $80 / 2010.1$ & 0.4217 & 2.1576 & 5.2597 & 1.1430 & 0.5075 & 3.5524 & 8.5560 & 1.8804 & 8 & 0.3376 & 2.0462 & 5.1654 & 1.3871 \\
\hline 50/50 Undyed & 0.5915 & 3.2731 & 7.3673 & 1.8316 & 0.4269 & 3.7817 & 10.5978 & 2.6314 & 9 & 0.6935 & 1.6405 & 3.3258 & 0.6442 \\
\hline $50 / 501.0$ & 0.0482 & 0.9490 & 2.5423 & 0.5879 & 0.0493 & 0.9819 & 2.8499 & 0.5699 & 10 & 0.1929 & 2.4751 & 4.6457 & 1.0339 \\
\hline $50 / 506.0$ & 0.2414 & 1.3181 & 3.2488 & 0.6965 & 0.2464 & 1.6315 & 3.9453 & 0.6812 & & & & & \\
\hline $50 / 5010.1$ & 0.3479 & 1.8137 & 3.9260 & 0.9116 & 0.2710 & 2.1088 & 4.8555 & 1.1190 & & & & & \\
\hline 20/80 Undyed & 0.4218 & 3.1963 & 8.6219 & 1.9096 & 0.3793 & 3.1337 & 9.2004 & 1.9321 & & & & & \\
\hline $20 / 801.0$ & 0.0577 & 0.8384 & 2.3662 & 0.6358 & 0.0679 & 0.9353 & 2.5658 & 0.5215 & & & & & \\
\hline $20 / 806.0$ & 0.2968 & 1.3929 & 3.4626 & 0.8340 & 0.2463 & 1.5261 & 3.5298 & 0.8123 & & & & & \\
\hline $20 / 8010.1$ & 0.3263 & 2.3530 & 5.6714 & 1.3961 & 0.3130 & 2.4721 & 6.7046 & 1.2327 & & & & & \\
\hline Brown Undyed & 0.2169 & 1.4143 & 2.7410 & 0.7594 & 0.1449 & 1.5116 & 3.0096 & 0.7508 & & & & & \\
\hline Brown 1.0 & 0.0864 & 0.7496 & 1.7178 & 0.4625 & 0.1216 & 1.0210 & 2.3350 & 0.5938 & & & & & \\
\hline Brown 6.0 & 0.1684 & 1.1269 & 2.7709 & 0.6917 & 0.1791 & 1.1869 & 3.4394 & 0.6473 & & & & & \\
\hline Brown 10.1 & 0.2740 & 1.3069 & 2.8087 & 0.6227 & 0.3021 & 1.3073 & 3.0073 & 0.5592 & & & & & \\
\hline
\end{tabular}

\section{DISCUSSION}

In order to evaluate the reliability of spectral measurements within the field of hair coloring quality control, we tested both dyed and undyed swatches for their intrinsic variability. Before this investigation, we tried to assess the degree of unwanted light chaotic bouncing and scatter in human hairs.

Analyzing spectra of human hair swatches, we found that measurements including (SCI) and excluding (SCE) the diffused specular component resulted in a negligible difference. Human hairs do appear to sheen when hit by light, but the combination of their microscopic structure and macroscopic spatial arrangement makes it so that the human eye cannot perceive a uniform gloss, unless appropriate cosmetic treatments are applied. Nylon swatches, on the other hand, show a greater difference, with $\Delta$ Es increasing towards lighter shade dyes.

We also tested human hair swatches for macroscopic differences in spatial arrangements by sampling them lengthwise and in crossed dispositions. The comparison suggests, as expected, an influence on the measuring outputs of the hair strands slant within the field of view. 
We finally tested hair measured color variability in two different contexts. The intra-swatch case accounts for the variance of a single swatch and represents a hypothetical two-point analysis of color homogeneity after a dyeing process. The inter-swatch case accounts for color reproducibility between pairs of swatch samples, one of the two being for instance a master swatch to be used as a reference. Both cases show similar behaviors.

Undyed versions of swatches show a twofold result. On one hand, Yak and Brown result sufficiently homogeneous, while 80/20, 50/50 and 20/80 (all containing a different mixture of pigmented and unpigmented hair strands) show greater variance, with mean $\Delta$ Es way higher than those associated with the minimum perceivable difference. $\Delta$ Es are consistently lower for Black (1.0) on all swatches, which appear homogeneous. From these values, $\Delta$ Es increase for Cold Dark Blond (6.0), and again for Ash Platinum Blond (10.1). These effects are less evident on Brown swatches. A possible explanation is the fact that the combination of the dye and the oxidizing agent did not possess a sufficiently strong depigmentation capability for the base color to be significantly altered. Nylon swatches, for which only intra-swatch measurements are available, also show a steady increment in $\Delta$ Es.

\section{CONCLUSIONS}

The artificial alteration of human hair is usually fraught with intricacies that might impinge on the final result. For the assessment of product quality and reproducibility, visual inspection is still preferred, though some dye makers resort to spectrophotometric measurements. However, given that human hairs are very efficient light scatterers, quantitative results may be too volatile for comfort. We would advise against uncritical confidence in numbers, and instead urge caution until more reliable figures of merit, possibly revolving around human perception, are introduced.

\section{REFERENCES}

[1] Bohren CF, Huffman DR. Absorption and scattering of light by small particles. John Wiley \& Sons; 2008.

[2] Rees JL. Genetics of Hair and Skin Color. Annual Review of Genetics. 2003;37(1):67-90. doi:10.1146/annurev.genet.37.110801.143233

[3] da França S, Dario M, Esteves V, Baby A, Velasco M. Types of Hair Dye and Their Mechanisms of Action. Cosmetics. 2015;2(2):110-126. doi:10.3390/cosmetics2020110

[4] Vaughn M, van Oorschot R, Baindur-Hudson S. Hair color measurement and variation. American Journal of Physical Anthropology. 2008;137(1):91-96. doi:10.1002/ajpa.20849

\section{AUTHORS BIOGRAPHIES}

Simone Liberini received his Bachelor and Master of Science degrees in Biomedical Engineering at Politecnico di Milano. He briefly worked as a research fellow at Istituto di Bioimmagini e Fisiologia Molecolare, Milano, where he contributed to developing a quantitative image-based frame of assessment of parotid glands shrinkage in head-and-neck cancer patients. He is currently fellow frequenter at Università degli Studi di Milano.

Antonio Avolio is a pharmaceutical chemist, researcher and cosmetic products formulator. Lecturer of trichology and cosmetics master courses at Università della Calabria (Italy) and Università di Ferrara (Italy), he collaborated with numerous companies active in the dermatological sector, and authored a number of patents both of instrumentations and innovative formulations. He's currently head of UBP Research Laboratories, NG Group, Brescia, Italy.

Stevn Thomas Yankowski is an award winning visual communications specialist coming from a diversified background as hairdresser / makeup artist, photographer, stylist, graphic artist, with expertise in marketing, product and brand development with over 25 years of experience in the cosmetics industry.

Alessandro Rizzi is full professor, Department of Computer Science, University of Milano. He has been one of the founders of the Italian Color Group, secretary of CIE Division 8, an IS\&T Fellow and a past Vice-President. He is topical editor for Applied Color Science of Journal of Optical Society of America A and associate editor of Journal of Electronic Imaging. In 2015, he received the Davies medal from the Royal Photographic Society. 